\title{
Continuous Passive Motion Does Improve Range of Motion, Pain and Swelling After ACL Reconstruction: A Systematic Review and Meta-Analysis
}

\section{Kontinuierliche passive Bewegungsschiene verbessert den Bewegungsumfang, Schmerz und Schwellung bei ACL- Rekonstruktion: Eine systematische Literaturübersichtsarbeit und Metaanalyse}

Authors

Theo Jaspers ${ }^{1}$, Jan Taeymans ${ }^{2}$, Anja Hirschmüller ${ }^{3}$, Heiner Baur ${ }^{2}$, Roger Hilfiker $^{4}$, Slavko Rogan ${ }^{2,5}$

\section{Affiliations}

1 Therapien, Siloam Klinik, Bern, Switzerland

2 Departement Gesundheit, Berner Fachhochschule, Bern, Switzerland

3 Sport, Altius Swiss Sportmed Center AG, Rheinfelden, Switzerland

4 Institute of Health, University of Applied Sciences ValaisWallis, Leukerbad, Germany

5 Academy of Physiotherapy and Trainingseducation, Grenzach-Wyhlen, Germany

Key words

continuous passive motion therapy, anterior cruciate ligament reconstruction, rehabilitation

Schlüsselwörter

kontinuierliche passive Bewegungsbehandlungen, Rekonstruktion des vorderen Kreuzbands, Rehabilitation

\section{Bibliography}

DOI https://doi.org/10.1055/a-0710-5127

Published online I Z Orthop Unfall @ Georg Thieme Verlag KG Stuttgart · New York | ISSN 1864-6697

Correspondence

Dr. Slavko Rogan

Departement Gesundheit, Berner Fachhochschule

Murtenstrasse 10, 3008 Bern, Switzerland

Phone: $+41 / 31 / 8483556$

slavko.rogan@bfh.ch

\section{ABSTRACT}

Background This systematic review and meta-analysis evaluates the results of empirical studies on the effects of CPM on rehabilitation after $A C L$ reconstruction. The research question was: what are the effects of CPM on ROM, swelling and pain after $\mathrm{ACL}$ reconstruction?
Patients/Material and Methods We searched in MEDLINE, Embase, CINAHL, Cochrane and PEDro up to January 2018. Standardized mean differences (SMD) were expressed as Hedges' $\mathrm{g}$, in order to correct for overestimation of the true effect in small study samples. The $95 \%$ confidence intervals $(95 \% \mathrm{Cl})$ were calculated for both the individual studies and the overall weighted estimate. Outcomes were range of motion, pain and swelling.

Results Eight studies comprising 442 participants were included in the meta-analysis. Beneficial effects of CPM could be identified for the need for pain medication (Hedges' $g=0.93 ; 95 \% \mathrm{Cl}=0.41$ to 1.45 during the first 24 hours after surgery), the number of PCA button pushes by the patient during the first 24 hours after surgery $(M D=31.20$; $95 \%$ $\mathrm{Cl}=11.35$ to 51.05$)$, on regaining knee flexion on the third to the seventh postoperative day $\left(\mathrm{MD}=11.6^{\circ} ; 95 \% \mathrm{Cl}=1.96\right.$ to 21.33 ) as well as in the third to the sixth postoperative week (Hedges' $g=0.93 ; 95 \% \mathrm{Cl}=0.41$ to 1.44 ) and on swelling of the knee in the fourth to sixth postoperative week (Hedges' $g=0.77 ; 95 \% \mathrm{Cl}=0.35$ to 1.18 ).

Conclusion This meta-analysis suggests that CPM has beneficial effects on pain reduction during the first two postoperative days, on knee flexion during the first to the sixth postoperative weeks and on swelling between the fourth and the sixth postoperative weeks. However, the risk-of-bias scores do not allow a high level of evidence.

\section{ZUSAMMENFASSUNG}

Hintergrund Diese systematische Literaturübersichtsarbeit und Metaanalyse evaluierte die Ergebnisse aus empirischen Studien über die Auswirkungen von kontinuierlichen passiven Bewegungsbehandlungen (KPB) auf die Rehabilitation nach ACL-Rekonstruktion. Die Forschungsfrage war: Was sind die Auswirkungen von CPM auf ROM, Schwellungen und Schmerzen nach ACL-Rekonstruktion?

Patient/Material und Methoden Wir suchten in den Datenbanken CINAHL, Cochrane, Embase, MEDLINE, und PEDro 


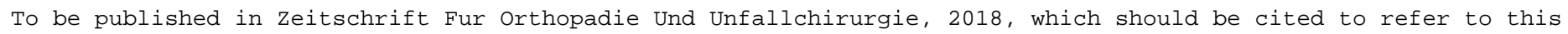
work, DOI:10.1055/a-0710-5127

bis Januar 2018. Standardisierte mittlere Differenzen (SMD) werden in Form von Hedges' $\mathrm{g}$ dargestellt, um eine Überschätzung der wahren Wirkung in kleinen Studienproben zu korrigieren. Für die einzelnen Studien als auch für die gewichtete Gesamtschätzung wurden 95\%-Konfidenzintervalle (95\%-KI) berechnet. Outcomes waren Beweglichkeit, Schmerzen und Schwellungen.

Ergebnisse Acht Studien mit 442 Probanden wurden in diese Metaanalyse eingeschlossen. Positive Effekte von KPB konnten in Bezug i) auf Schmerzmittelgabe während den ersten 24 Stunden nach der Operation (Hedges' $g=0,93$; 95\%$\mathrm{KI}=0,41-1,45)$, ii) auf PCA-Gabe vom Patienten während der ersten 24 Stunden nach der Operation (MD $=31,20$; 95\%-
$\mathrm{KI}=11,35-51,05)$, iii) nach wiedererlangter Knieflexionsbeweglichkeit am 3.-7. postoperativen $\operatorname{Tag}\left(\mathrm{MD}=11,6^{\circ}\right.$; $95 \%-K I=1,96-21,33)$ sowie in der 3.-6. postoperativen Woche (Hedges' $g=0,93 ; 95 \%-K I=0,41-1,44$ ) und iv) bei Schwellung des Knies in der 4.-6. postoperativen Woche (Hedges' $g=0,77 ; 95 \%-K I=0,35-1,18$ ) identifiziert werden.

Schlussfolgerung Diese Metaanalyse zeigt auf, dass KPB positive Auswirkungen auf die Schmerzreduktion in den ersten beiden postoperativen Tagen, auf Knieflexion während der 1.-6. postoperativen Woche und auf Schwellungen zwischen der 4. und 6. postoperativen Woche hat. Die Risk-of-BiasScores erlauben jedoch keine hohen Evidenzgrade.

\section{Background}

The application of continuous passive motion devices (CPM) has a firmly established role in standard postoperative treatment after surgical reconstruction of the anterior cruciate ligament $(A C L)$. Salter et al. [1-4] proposed and promoted the application of CPM devices after joint surgery or trauma in order to relieve the adverse effects of immobilization. Empirical investigation postulated that the objectives of CPM are to increase range of motion, reduce swelling, decrease pain, help prevent capsular contractures, and to improve the orientation and strength of collagen fibres in the ACL graft [5].

In 2007 Smith and Davies [6] conducted a systematic review about the effects of CPM following ACL reconstruction. They included eight papers, all published in English. They concluded that it remained unclear whether postoperative CPM has any benefit for patients following $A C L$ reconstructions and recommended further study in order to improve the evidence. In their discussion section Smith and Davies [6] stated that "further review assessing evidence published in other languages may be indicated to compare against the findings of this review". In 2012 Lobb, Tumilty and Claydon [7] published a qualitative synthesis of five systematic reviews on rehabilitation after $\mathrm{ACL}$ reconstruction. Concerning CPM, their conclusions were solely based on the previously mentioned review of Smith and Davies [6]. The authors also stated in their discussion section: "It is acknowledged that a language restriction was imposed on this review to RCTs in English, which may have introduced a language bias (Egger et al. [8]) [7]”. According to the Cochrane Handbook for Systematic Reviews of Interventions, language restrictions could introduce reporting bias and might influence the effect size of a meta-analysis [9]

So far, no review exists that

1. included studies published in languages other than English and

2. conducted a meta-analysis.

Therefore, the aim of this study was to conduct a systematic review and meta-analysis in order to summarise the results of empirical studies on the effects of CPM on rehabilitation after ACLreconstruction and to evaluate their variability.

The research question for this systematic review and metaanalysis is:
"What are the effects of continuous passive motion on range of motion, swelling and pain after ACL-reconstruction?"

\section{Patients/Material and Methods}

\section{Protocol and registration}

Methods of the analysis and inclusion criteria were specified in advance, documented in a protocol and published on the Prospero international prospective register of systematic reviews, registration number CRD42014015077. The systematic literature search, the analysis and synthesis were performed according to the PRISMA statement [13].

\section{Literature search strategies and data sources}

A literature search through the electronic databases of MEDLINE, Embase, CINAHL, Cochrane and PEDro was performed between November 2014 and November 2017 using the keywords "(continuous AND passive AND motion OR cPm) AND (anterior AND cruciate AND ligament OR acl)". The search strategy was reviewed by the qualified chief librarian of the University of Applied Sciences, Health, in Bern [10] as well as by a Doctor of Biology who is familiar with database searching [11].

Following the Preferred Reporting Items for Systematic Reviews and Meta-Analyses (PRISMA [12]), the corresponding PICOS chart is:

- Population: Patients after surgical Anterior Cruciate Ligament $(\mathrm{ACL})$ reconstruction

- Intervention: Rehabilitation with continuous passive motion (CPM)

- Comparator: Rehabilitation without CPM

- Outcomes: Range of motion and/or pain and/or swelling

- Study design: Randomised controlled trial, clinical trial or retrospective study

In order to find the maximum number of results, for the Cochrane database the keywords were changed to "continuous passive motion ACL". As an illustration, Box 2 in the Supplement section shows details of the database search strategy. No restrictions regarding publication year or language were imposed in order to yield the widest possible range of relevant papers. The resulting 
list was checked for completeness by an expert professor in orthopaedic surgery.

\section{Identification and study selection}

Inclusion criteria for the current systematic review were:

1. Study design: clinical trial or retrospective study,

2. Participants: patients after surgical $A C L$ reconstruction, male and female, all ages, all operation techniques and all transplantation tissues, all time lapse durations between injury and surgery,

3. Intervention: postoperative rehabilitation procedure comprising CPM, all devices and brands (Kinetec, Arthromot, Optiflex, Sutter, Stryker),

4. Comparison: no CPM, and

5. Outcome: Range of motion [13] and/or swelling and/or pain, expressed in any parameter.

Exclusion criteria were:

1. trials on tissue testing in laboratories or animal testing,

2. trials without control group,

3. trials with CPM in both intervention and control group,

4. neither ROM, nor swelling nor pain as an outcome parameter,

5. reviews or guidelines,

6. editorials and comments.

Two independent researchers (TJ, SR) conducted the selection procedure. Disagreements were discussed until consensus was reached.

\section{Data analysis}

Two authors (TJ, SR) independently extracted and cross-checked the data from the included studies. The following study characteristics were extracted: authors and year of publication, publication language, study design, number of subjects, details of interventions (both surgery and CPM application) as well as controls and outcome measures matching the research question. Disagreements were discussed until consensus was achieved.

Any missing information was requested from the corresponding authors by e-mail. If missing information about measures of statistical dispersion could no longer be retrieved, standard deviations were generated according to the Cochrane Handbook for Systematic Reviews of Interventions of Higgins and Green [14] as described in section "Methodological quality".

If studies reported $\mathrm{p}$-values to their reported means without any further measures of statistical dispersion and this was expressed as " $p<0.05$ " instead of the exact $p$-value, a conservative position was chosen by setting the $p$-value to 0.05 in the metaanalysis calculations.

\section{Risk-of-bias assessment}

All studies were critically appraised for methodological quality by two researchers independently (TJ, SR), using the Cochranes's risk-of-bias tool as described in the Cochrane Handbook for Systematic Reviews of Interventions [14]. Disagreements were resolved by discussion. Each study was graded for the following domains: sequence generation; allocation concealment; blinding participants, personnel, assessors; incomplete outcome data; selective outcome reporting; other sources of bias. These were rated low or high. In case of insufficient information, the risk-ofbias item was rated "unclear".

\section{Data synthesis and statistical analysis}

All available outcomes were organised in order to establish comparability and eligibility for the systematic review. Two time frames were defined, the first-time frame covering the short-term period of the first seven postoperative days, and the second referring to the long-term period of the second to the sixth postoperative week s. If outcome data of at least two studies were found regarding either ROM or swelling or pain within the defined time frames, these data were preselected for the meta-analysis. If several measurement time points within the same time frame were found in the same study, the outcome referring to the latest time point within this time frame was selected.

An a priori specified random-effects model was used in order to examine the overall effect size of CPM on ROM, swelling and on pain-related outcomes. If outcome data resulted from identical measurement procedures and were expressed in the same unit across all studies included, effect measures were calculated as differences in means (MD), intending to provide information that allows easy interpretation by clinicians. For those outcomes resulting from measurement procedures that were not identical or were not expressed in the same unit across all studies, effect measures were calculated as standardised mean differences (SMD) and expressed as Hedges' $g$ in order to correct for overestimation of the true effect in small study samples. The $95 \%$ confidence intervals $(95 \% \mathrm{Cl})$ were calculated for both the individual studies and the overall weighted estimate.

Where single studies showed outlying values, sensitivity analyses were conducted in order to examine the influence of these studies on the overall weighted effect sizes.

To test for heterogeneity across studies, Q-values and the corresponding degrees of freedom and p-value were calculated. To assess how much of the total variance can be explained by the variability between studies, $\mathrm{I}^{2}$ was calculated and interpreted as $<25 \%=$ indicates small, $>50 \%=$ indicates moderate and $>75 \%$ indicates considerable heterogeneity, according to Higgins et al. [15].

For those outcomes providing sufficient data for pooling, publication bias was assessed using a funnel plot. The "fail 'n safe" algorithm was used to assess how many non-significant studies would be needed to bring the overall weighted mean to the alpha level while the trim and fill algorithm was used to test how robust the overall weighted mean was against funnel plot asymmetry.

For all analyses, $\mathrm{p}$-values less than 0.05 were considered significant. The meta-analyses were performed using the CMA-3 metaanalysis software, version 3.3.070 (Biostat Inc., Englewood USA).

\section{Results}

\section{Flow of studies through the review}

After elimination of duplicates, a total of 100 articles resulted from the literature search. 19 articles met the a priori set inclusion criteria and were read in detail. A professional translation agency 


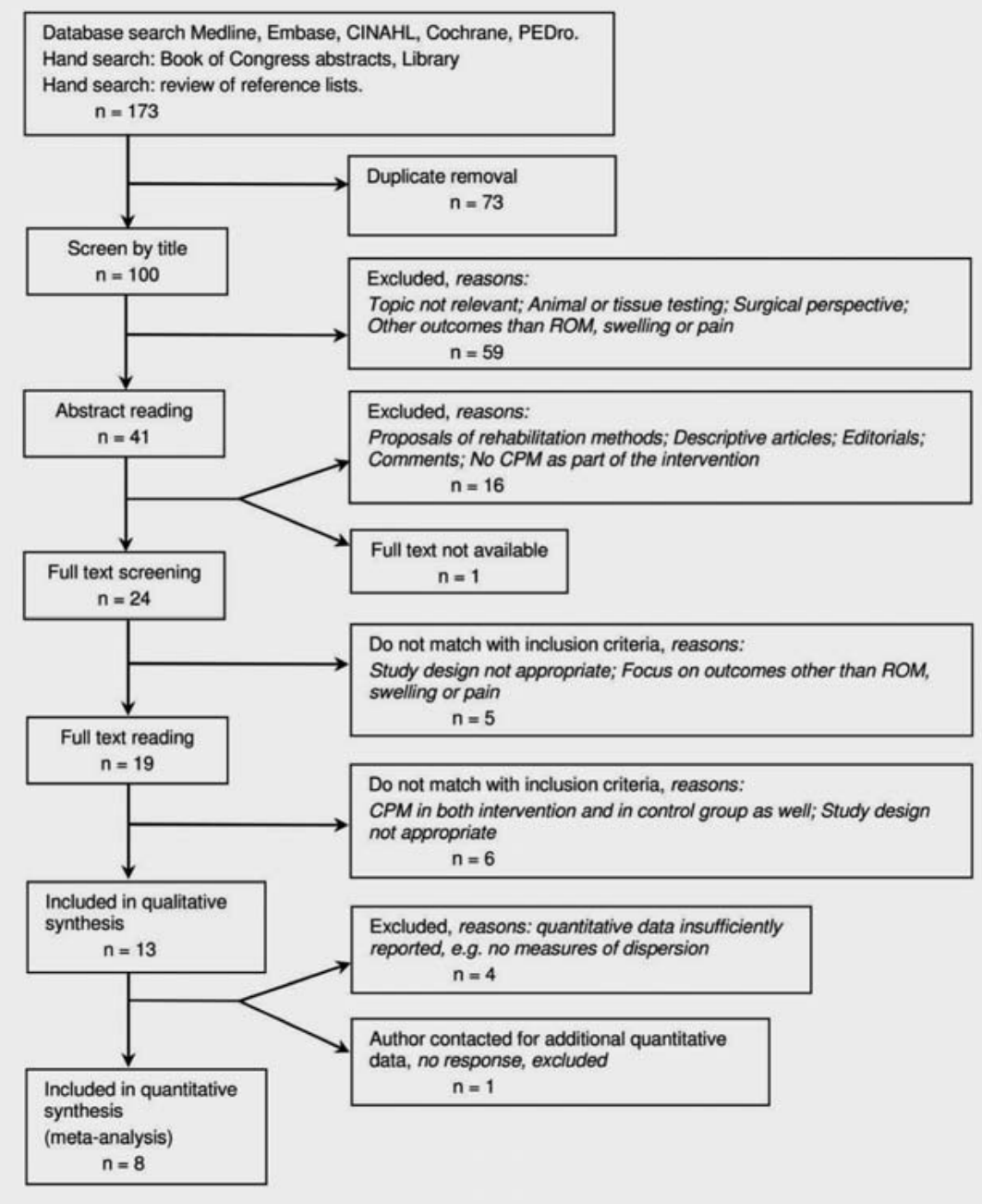

- Fig. 1 Flowchart of study selection procedure.

was engaged to translate one Turkish publication [16] into English. One paper [17] was excluded on the account of insufficient data. Eight studies $[13,16,18-23]$ were finally included in the meta-analysis. The selection procedure setting out reasons for exclusion is shown as a flowchart in • Fig. $\mathbf{1}$.

\section{Study characteristics}

The eight articles included were published between 1987 and 2013 and were conducted in Australia $(n=1)$ [22], Italy $(n=1)$ [21], Sweden $(n=1)$ [13], Switzerland $(n=1)$ [18], Turkey $(n=1)$ [16], and the USA $(n=3)[19,20,23]$. The total study population of all selected articles together comprised 442 participants, of whom 156 had been assessed for differences in range of motion in five studies $[13,16,18,20,23], 122$ for differences in swelling in four studies $[13,20,21,23]$ and 336 for differences in pain in four studies $[16,19,22,23]$. All participants underwent arthroscopic ACL reconstruction using semitendinosus or patellar ligament tendon grafts, with or without meniscal repair. There were five randomised controlled trials $[13,18-20,23]$, two clinical trials [21,22] and one retrospective study [16].
All participants in the intervention groups received CPM after surgery. However, the onset of CPM varied from immediately after surgery $[22,23]$ to starting on the third postoperative day [16]. The end of the CPM intervention also varied. The shortest CPM application lasted until after the first postoperative day [22]. The longest application lasted throughout the hospital stay $[13,16$, 17, 20-22]. If specified, most CPM devices were set to a maximum of $60^{\circ}$ of flexion at the beginning, gradually increasing up to $90^{\circ}$ if tolerated by the patient $[16,19-21,23]$. The dosage per day varied from a minimum of one hour daily [16] to 24 hours daily [22].

Range of motion in flexion was expressed in degrees measured by a customary goniometer as absolute values $[16,18,20,23]$ or as flexion deficit compared to the non-operated knee [13]. Extension was expressed in degrees measured by goniometer as absolute values [16, 20], as extension deficit [18] or as extension deficit compared to the non-operated knee [13].

Swelling was expressed in differences in cm circumference compared to the preoperative values $[20,21]$ or in comparison to the sound leg [13], measured at upper-patella level or above as 
- Table 1 Detailed study characteristics.

\begin{tabular}{|c|c|c|c|c|c|c|}
\hline $\begin{array}{l}\text { Study } \\
\text { Language }\end{array}$ & Design & Participants & $\begin{array}{l}\text { Intervention } \\
\text { control }\end{array}$ & \multicolumn{3}{|c|}{ Outcome measures } \\
\hline \multirow{18}{*}{$\begin{array}{l}\text { Bizzini et al. } \\
\text { [18] } \\
\text { German }\end{array}$} & \multirow[t]{18}{*}{ RCT } & \multirow{18}{*}{$\begin{array}{l}\mathrm{n}=14 \\
\text { Isolated reconstruction } \\
\text { of anterior cruciate liga- } \\
\text { ment by semitendinosus } \\
\text { tendon or patellar liga- } \\
\text { ment and meniscal repair } \\
\text { (first time) }\end{array}$} & \multirow{18}{*}{$\begin{array}{l}\text { Int: }>6-8 \text { hours } \\
\text { CPM daily } \\
\text { Con: } 4 \times 30^{\prime} \text { daily } \\
\text { CAM }\end{array}$} & \multicolumn{3}{|l|}{ ROM: Flexion } \\
\hline & & & & \multicolumn{3}{|c|}{$\begin{array}{l}\text { Passive knee flexion in degrees (interquartile range) measured by } \\
\text { goniometer on: }\end{array}$} \\
\hline & & & & Postop day 1 & CPM: 60 (18.8) & Con: $30(25.0)$ \\
\hline & & & & Postop day 2 & CPM: 80 (10.0) & Con: $50(28.8)$ \\
\hline & & & & Postop day 3 & CPM: 90 (12.5) & Con: 60 (18.8) \\
\hline & & & & Postop day 4 & CPM: 95 (8.8) & Con: 70 (13.8) \\
\hline & & & & Postop day 5 & CPM: 100 (3.8) & Con: 80 (13.8) \\
\hline & & & & Postop day 8 & CPM: 105 (12.5) & Con: 90 (20.0) \\
\hline & & & & Postop 1 month & CPM: 125 (12.5) & Con: $110(17.5)$ \\
\hline & & & & \multicolumn{3}{|l|}{ ROM: Extension } \\
\hline & & & & \multicolumn{3}{|c|}{$\begin{array}{l}\text { Knee extension deficit in degrees (interquartile range) measured by } \\
\text { goniometer on: }\end{array}$} \\
\hline & & & & Postop day 1 & CPM: 0.0 (10.0) & Con: $10.0(16.3)$ \\
\hline & & & & Postop day 2 & CPM: 0.0 (3.8) & Con: $10.0(12.5)$ \\
\hline & & & & Postop day 3 & CPM: $5.0(8.8)$ & Con: $5.0(10.0)$ \\
\hline & & & & Postop day 4 & CPM: 5.0 (5.5) & Con: $5.0(8.8)$ \\
\hline & & & & Postop day 5 & CPM: $5.0(5.0)$ & Con: $5.0(5.0)$ \\
\hline & & & & Postop day 8 & CPM: 0.0 (3.8) & Con: $5.0(5.0)$ \\
\hline & & & & Postop 1 month & CPM: $0.0(0.0)$ & Con: $0.0(0.0)$ \\
\hline \multirow{12}{*}{$\begin{array}{l}\text { Engstrom } \\
\text { et al. [13] } \\
\text { English }\end{array}$} & \multirow[t]{12}{*}{ RCT } & \multirow{12}{*}{$\begin{array}{l}\mathrm{n}=34 \\
\text { Unilateral arthroscopic } \\
\text { ACL reconstruction with } \\
\text { patella tendon grafts }\end{array}$} & \multirow{4}{*}{$\begin{array}{l}\text { Int: active dy- } \\
\text { namic flexion } 1 \times \\
\text { daily, supervised } \\
\text { by physiothera- } \\
\text { pist, + } 6 \text { hours } \\
\text { CPM daily }\end{array}$} & \multicolumn{3}{|l|}{ ROM: Flexion } \\
\hline & & & & \multicolumn{3}{|c|}{$\begin{array}{l}\text { Passive knee flexion deficit in degrees (standard deviation), compared to the } \\
\text { other leg (side-to-side difference), measured by goniometer on: }\end{array}$} \\
\hline & & & & Postop 6 weeks & CPM: 22.1 (21.4) & Con: $26.9(17.1)$ \\
\hline & & & & \multicolumn{3}{|l|}{ ROM: Extension } \\
\hline & & & Con: active dy- & \multicolumn{3}{|c|}{$\begin{array}{l}\text { Passive knee extension deficit in degrees (standard deviation), compared to } \\
\text { the other leg (side-to-side difference), measured by goniometer on: }\end{array}$} \\
\hline & & & daily, supervised & Postop 6 weeks & CPM: 3.0 (3.7) & Con: $3.6(3.4)$ \\
\hline & & & by physiothera- & \multicolumn{3}{|l|}{ Swelling: } \\
\hline & & & $\begin{array}{l}\text { pist, full exten- } \\
\text { sion allowed in }\end{array}$ & \multicolumn{3}{|c|}{$\begin{array}{l}\text { Midpatellar circumference in } \mathrm{cm} \text { (standard deviation), compared to the } \\
\text { other leg (side-to- side difference), measured on: }\end{array}$} \\
\hline & & & & Postop 6 weeks & CPM: $1.0(1.0)$ & Con: $1.6(0.6)$ \\
\hline & & & & \multicolumn{3}{|l|}{ Swelling: } \\
\hline & & & & \multicolumn{3}{|c|}{$\begin{array}{l}\text { Base patella circumference in cm (standard deviation), compared to the } \\
\text { other leg (side- to-side difference), measured on: }\end{array}$} \\
\hline & & & & Postop 6 weeks & CPM: $1.0(1.0)$ & Con: $1.6(0.6)$ \\
\hline
\end{tabular}

continued next page

well as mid-patella $[13,20,21]$. In one study, swelling was expressed by a cross-sectional area measurement in $\mathrm{cm}^{2}$ at lowerpatella level, above-patella and mid-patella [23].

Pain was expressed in $\mathrm{mg}$ pain medication (patient-controlled analgesia (PCA) [19,23], oral [19,22] or intramuscular [22]), number of PCA button pushes by the patient [19,23], score on a visual analogue scale [16] or as a score on the Perceived Pain Score questionnaire [19]. Details of the study characteristics are displayed in - Table 1.

\section{Methodological quality}

The quality assessment according to the "Cochrane Collaboration's risk-of-bias tool" [14] showed that most of the studies provided insufficient information about randomisation and blinding procedures. As a result, for many studies the items "sequence generation" [13,16,19-22], "allocation concealment" [13,16, $18-23]$ and "blinding participants, personnel, assessors" [13, 16, $18,20,23]$ remained predominantly unclear. Three studies reported incomplete data $[16,18,21]$, one study reported selec- 
- Table 1 Detailed study characteristics. continued

\begin{tabular}{|c|c|c|c|c|c|c|}
\hline $\begin{array}{l}\text { Study } \\
\text { Language }\end{array}$ & Design & Participants & $\begin{array}{l}\text { Intervention } \\
\text { control }\end{array}$ & \multicolumn{3}{|l|}{ Outcome measures } \\
\hline \multirow{24}{*}{$\begin{array}{l}\text { McCarthy } \\
\text { et al. [19] } \\
\text { English }\end{array}$} & \multirow[t]{24}{*}{ RCT } & \multirow{24}{*}{$\begin{array}{l}\mathrm{n}=30 \\
\text { Arthroscopic ACL recon- } \\
\text { struction with bone- } \\
\text { patella tendon-bone } \\
\text { autografts }\end{array}$} & \multirow{24}{*}{$\begin{array}{l}\text { Int: rehab pro- } \\
\text { gramme with } \\
\text { CPM } 16 \text { hours } \\
\text { daily for } 3 \text { days, } \\
\text { flexion at least } \\
60^{\circ} \text {, increased by } \\
\text { patient to } 90^{\circ} \text { as } \\
\text { tolerated } \\
\text { Con: identical re- } \\
\text { hab programme } \\
\text { but without CPM }\end{array}$} & \multicolumn{3}{|l|}{ Pain: } \\
\hline & & & & \multicolumn{3}{|c|}{ Medication via PCA pump in mg (standard deviation) } \\
\hline & & & & $\begin{array}{l}\text { During first } 24 \text { hrs } \\
\text { postop: }\end{array}$ & CPM: 41 (18.9) & Con: $65(21.4)$ \\
\hline & & & & \multicolumn{3}{|l|}{ Pain: } \\
\hline & & & & \multicolumn{3}{|c|}{ Number of times patient pushed PCA button (standard deviation) } \\
\hline & & & & $\begin{array}{l}\text { During first } 24 \mathrm{hrs} \\
\text { postop: }\end{array}$ & CPM: 30 (16.3) & Con: $62(51.3)$ \\
\hline & & & & \multicolumn{3}{|l|}{ Pain: } \\
\hline & & & & \multicolumn{3}{|c|}{ Oral medication in number of tablets (standard deviation) } \\
\hline & & & & $\begin{array}{l}\text { During postop days } \\
2+3 \text { : }\end{array}$ & CPM: $10(2.7)$ & Con: $14(6.9)$ \\
\hline & & & & \multicolumn{3}{|l|}{ Pain: } \\
\hline & & & & \multicolumn{3}{|c|}{ Perceived Pain Score, question 1 (standard deviation): } \\
\hline & & & & On postop day 1: & CPM: $5.6(2.1)$ & Con: $6.2(2.7)$ \\
\hline & & & & On postop day $2:$ & CPM: $2.9(2.0)$ & Con: 4.5 (2.8) \\
\hline & & & & On postop day $3:$ & CPM: 2.5 (1.8) & Con: $2.9(1.9)$ \\
\hline & & & & \multicolumn{3}{|l|}{ Pain: } \\
\hline & & & & \multicolumn{3}{|c|}{ Perceived Pain Score, question 2 (standard deviation): } \\
\hline & & & & On postop day 1 : & CPM: $8.6(2.0)$ & Con: $9.3(2.0)$ \\
\hline & & & & On postop day 2: & CPM: 5.8 (3.3) & Con: 7.5 (2.9) \\
\hline & & & & On postop day 3: & CPM: $4.2(2.7)$ & Con: $6.1(3.1)$ \\
\hline & & & & \multicolumn{3}{|l|}{ Pain: } \\
\hline & & & & \multicolumn{3}{|c|}{ Perceived Pain Score, question 3 (standard deviation): } \\
\hline & & & & On postop day 1 : & CPM: $2.0(1.5)$ & Con: $2.4(1.6)$ \\
\hline & & & & On postop day 2: & CPM: 1.1 (1.0) & Con: $1.4(1.6)$ \\
\hline & & & & On postop day 3: & CPM: $0.9(0.8)$ & Con: 0.8 (0.6) \\
\hline
\end{tabular}

continued next page

tively [21] and four studies showed other sources of bias $[13,16$, $20,22]$. The details of the risk-of-bias analysis of the individual studies can be observed in > Table 2 .

According to the Cochrane Handbook for Systematic Reviews of Interventions of Higgins and Green [14] the reported interquartile ranges (IQR) in Bizzini et al. [18] had to be transformed into standard deviations (SD), calculating $S D=I Q R / 1.35$.

In Rigon et al. [21] a missing standard deviation had to be replaced by a plausible value, derived from another study with the same outcome, following the method described in the Cochrane Handbook for Systematic Reviews of Interventions [14]. A sensitivity analysis was conducted in order to investigate the effect of this action, as specified in the Discussion section.

\section{Effects of continuous passive motion}

Range of motion, flexion in degrees during the third to seventh postoperative days: CPM showed significantly higher flexion values during the third to seventh postoperative days $\left(\mathrm{MD}=11.6^{\circ}\right.$ flexion; $95 \% \mathrm{Cl}=1.96$ to $21.33 ; \mathrm{Q}=8.40 ; \mathrm{df}(\mathrm{Q})=3 ; \mathrm{p}=0.04$;
$I^{2}=64 \%$ ) when compared with rehabilitation without CPM, as shown in > Fig. 2.

Range of motion, flexion during the third to sixth postoperative weeks: CPM showed significantly higher flexion values during the third to sixth postoperative weeks (Hedges' $g=0.93 ; 95 \%$ $\mathrm{Cl}=0.41$ to $1.44 ; \mathrm{Q}=9.12 ; \mathrm{df}(\mathrm{Q})=4 ; \mathrm{P}=0.06 ; \mathrm{I}^{2}=56 \%$ ) compared with rehabilitation without CPM as shown in > Fig. 3 .

Range of motion, extension during the third to seventh postoperative days: CPM showed no significant effect on extension values during the third to seventh postoperative days (Hedges' $\mathrm{g}=0.09 ; 95 \% \mathrm{Cl}=-0.31$ to $0.49 ; \mathrm{Q}=0.51 ; \mathrm{df}(\mathrm{Q})=2 ; \mathrm{p}=0.78$; $\mathrm{I}^{2}=0 \%$ ) when compared with rehabilitation without CPM, as shown in > Fig. 4.

Range of motion, extension during the third to sixth postoperative weeks: CPM showed no significant effect on extension values during the third to sixth postoperative weeks (Hedges' $g=0.11$; $95 \% \mathrm{Cl}=-0.26$ to $0.47 ; \mathrm{Q}=0.48 ; \mathrm{df}(\mathrm{Q})=2$;

$\left.\mathrm{P}=0.79 ; \mathrm{I}^{2}=0 \%\right)$ when compared with rehabilitation without CPM, as shown in • Fig. 5. 
- Table 1 Detailed study characteristics. continued

\begin{tabular}{|c|c|c|c|c|c|c|}
\hline $\begin{array}{l}\text { Study } \\
\text { Language }\end{array}$ & Design & Participants & $\begin{array}{l}\text { Intervention } \\
\text { control }\end{array}$ & \multicolumn{3}{|c|}{ Outcome measures } \\
\hline \multirow{28}{*}{$\begin{array}{l}\text { Noyes et al. } \\
{[20]} \\
\text { English }\end{array}$} & \multirow[t]{28}{*}{ RCT } & \multirow{28}{*}{$\begin{array}{l}\mathrm{n}=18 \\
\text { Arthroscopic ACL repair/ } \\
\text { reconstruction }\end{array}$} & \multirow{28}{*}{$\begin{array}{l}\text { Int: rehab pro- } \\
\text { gramme with } \\
\text { CPM } 10 \text { hours } \\
\text { daily starting on } \\
\text { postop day } 1 \text {, in- } \\
\text { creasing until } 90^{\circ} \\
\text { Con: identical re- } \\
\text { hab programme } \\
\text { but without CPM }\end{array}$} & \multicolumn{3}{|l|}{ ROM: Flexion } \\
\hline & & & & \multicolumn{3}{|c|}{ Passive knee flexion in degrees (standard deviation) measured on: } \\
\hline & & & & Postop day 7 & CPM: $68(12)$ & Con: 63 (14) \\
\hline & & & & Postop 2 weeks & CPM: 81 (18) & Con: 70 (10) \\
\hline & & & & Postop 3 weeks & CPM: 93 (18) & Con: 73 (15) \\
\hline & & & & Postop 4 weeks & CPM: $102(17)$ & Con: $88(11)$ \\
\hline & & & & Postop 3 months & CPM: 120 (16) & Con: 117 (5) \\
\hline & & & & \multicolumn{3}{|l|}{ ROM: Extension } \\
\hline & & & & \multicolumn{3}{|c|}{ Passive knee extension in degrees (standard deviation) measured on: } \\
\hline & & & & Postop day 7 & CPM: 11 (8) & Con: 14 (7) \\
\hline & & & & Postop 2 weeks & CPM: $6(7)$ & Con: $16(9)$ \\
\hline & & & & Postop 3 weeks & CPM: 8 (15) & Con: 14 (6) \\
\hline & & & & Postop 4 weeks & CPM: 5 (6) & Con: 7 (5) \\
\hline & & & & Postop 3 months & CPM: $0(0)$ & Con: $2(4)$ \\
\hline & & & & \multicolumn{3}{|l|}{ Swelling: } \\
\hline & & & & \multicolumn{3}{|c|}{$\begin{array}{l}\text { Midpatellar circumference differences in cm (standard deviation), compared } \\
\text { to preoperative measurement, measured on: }\end{array}$} \\
\hline & & & & Postop day 2: & CPM: 2.4 (1.9) & Con: $3(1.0)$ \\
\hline & & & & Postop day 7: & CPM: 2.1 (1.6) & Con: $2(1.3)$ \\
\hline & & & & Postop 2 weeks: & CPM: $0.7(0.46)$ & Con: $0.9(0.81)$ \\
\hline & & & & Postop 3 weeks: & CPM: 0.6 (0.56) & Con: 1 (0.86) \\
\hline & & & & Postop 4 weeks: & CPM: $0.5(0.71)$ & Con: $0.9(0.64)$ \\
\hline & & & & \multicolumn{3}{|l|}{ Swelling: } \\
\hline & & & & \multicolumn{3}{|c|}{$\begin{array}{l}\text { Superior pole patella circumference differences in } \mathrm{cm} \text { (standard deviation), } \\
\text { compared to preoperative measurement, measured on: }\end{array}$} \\
\hline & & & & Postop day 2: & CPM: 2.7 (2.1) & Con: $2.6(1.7)$ \\
\hline & & & & Postop day 7: & CPM: 1.7 (2.0) & Con: $1.8(1.8)$ \\
\hline & & & & Postop 2 weeks: & CPM: $0.2(0.38)$ & Con: 0.3 (0.79) \\
\hline & & & & Postop 3 weeks: & CPM: - 0.9 (0.59) & Con: 0.3 (0.59) \\
\hline & & & & Postop 4 weeks: & CPM: - 0.7 (0.94) & Con: $0.2(0.92)$ \\
\hline \multirow{10}{*}{$\begin{array}{l}\text { Rigon et al. } \\
\text { [21] } \\
\text { English/Ita- } \\
\text { lian }\end{array}$} & \multirow{10}{*}{$\begin{array}{l}\text { Clinical tri- } \\
\text { al }\end{array}$} & \multirow{10}{*}{$\begin{array}{l}\mathrm{n}=40 \\
\mathrm{ACL} \text { reconstruction }\end{array}$} & \multirow{10}{*}{$\begin{array}{l}\text { Int: CPM } 1 \text { hour } \\
3 \times \text { daily starting } \\
\text { on postop day } 1 \text {, } \\
\text { initially } 60^{\circ} \text {, in- } \\
\text { creasing } 10^{\circ} \text { daily. } \\
\text { Con: active or } \\
\text { assisted-active } \\
\text { flexion/extension } \\
\text { exercises }\end{array}$} & \multicolumn{3}{|l|}{ Swelling: } \\
\hline & & & & \multicolumn{3}{|c|}{$\begin{array}{l}\text { Midpatellar circumference differences in } \mathrm{cm} \text { (range), compared to preoper- } \\
\text { ative measurement, measured on: }\end{array}$} \\
\hline & & & & Postop day 5: & CPM: + $3.4(+2 /+5)$ & Con: + $3.1(0 /+5)$ \\
\hline & & & & Postop 2 weeks: & CPM: + $0.85(0 /+3)$ & Con: + $1.85(0 /+3.5)$ \\
\hline & & & & Postop 6 weeks: & CPM: + $0.75(0 /+4)$ & Con: + $1.45(0 /+4)$ \\
\hline & & & & \multicolumn{3}{|l|}{ Swelling: } \\
\hline & & & & \multicolumn{3}{|c|}{$\begin{array}{l}2 \mathrm{~cm} \text { above superior pole patella circumference differences in } \mathrm{cm} \text { (range), } \\
\text { compared to preoperative measurement, measured on: }\end{array}$} \\
\hline & & & & Postop day 5: & CPM: + $3.8(+2 /+5)$ & Con: + $3.3(0 /+4)$ \\
\hline & & & & Postop 2 weeks: & CPM: + $1.4(0 /+5)$ & Con: + $1.7(0 /+4)$ \\
\hline & & & & Postop 6 weeks: & CPM: + $0.9(0 /+2)$ & Con: + $1.2(0 /+3)$ \\
\hline
\end{tabular}


- Table 1 Detailed study characteristics. continued

\begin{tabular}{|c|c|c|c|c|c|c|c|}
\hline $\begin{array}{l}\text { Study } \\
\text { Language }\end{array}$ & Design & Participants & $\begin{array}{l}\text { Intervention } \\
\text { control }\end{array}$ & \multicolumn{4}{|l|}{ Outcome measures } \\
\hline \multirow{13}{*}{$\begin{array}{l}\text { Terzi et al. } \\
{[16]} \\
\text { Turkish }\end{array}$} & \multirow{13}{*}{$\begin{array}{l}\text { Retro- } \\
\text { spective } \\
\text { study }\end{array}$} & \multirow{13}{*}{$\begin{array}{l}\mathrm{n}=60 \\
\text { ACL reconstruction with } \\
\text { Hamstrings graft }\end{array}$} & \multirow{8}{*}{$\begin{array}{l}\text { Int: rehab pro- } \\
\text { gramme with } \\
\text { CPM starting on } \\
\text { postop day } 3 \text {, } \\
\text { flexion initially } \\
30-40^{\circ} \text {, in- } \\
\text { creased as toler- } \\
\text { ated, } 1 \text { hour a } \\
\text { day, for max. } \\
10 \text { days. }\end{array}$} & \multicolumn{4}{|l|}{ ROM: Flexion } \\
\hline & & & & \multicolumn{4}{|c|}{ Passive knee flexion in degrees (standard deviation) measured on: } \\
\hline & & & & Postop day 3 & CPM: 48.33 (21.10) & \multicolumn{2}{|c|}{ Con: 45.17 (21.6) } \\
\hline & & & & Postop 4 weeks & CPM: $108.83(15.24)$ & \multicolumn{2}{|c|}{ Con: $83.5(17.28)$} \\
\hline & & & & Postop 3 months & CPM: 119.67 (7.54) & \multicolumn{2}{|c|}{ Con: $112.83(11.72)$} \\
\hline & & & & \multicolumn{4}{|l|}{ ROM: Extension } \\
\hline & & & & \multicolumn{4}{|c|}{ Passive knee extension in degrees (standard deviation) measured on: } \\
\hline & & & & Postop day 3 & CPM: $5.50(6.34)$ & \multicolumn{2}{|c|}{ Con: 5.67 (6.79) } \\
\hline & & & \multirow{5}{*}{$\begin{array}{l}\text { Con: rehab pro- } \\
\text { gramme without } \\
\text { CPM }\end{array}$} & Postop 4 weeks & CPM: $1.17(2.52)$ & \multicolumn{2}{|c|}{ Con: $1.17(2.52)$} \\
\hline & & & & Postop 3 months & CPM: $1.33(2.92)$ & \multicolumn{2}{|c|}{ Con: $0.33(1.27)$} \\
\hline & & & & \multicolumn{4}{|l|}{ Pain: } \\
\hline & & & & \multicolumn{4}{|c|}{ Visual analogue scale (standard deviation), measured on: } \\
\hline & & & & Postop day 3 & CPM: $5.50(6.34)$ & \multicolumn{2}{|c|}{ Con: 5.67 (6.79) } \\
\hline \multirow{6}{*}{$\begin{array}{l}\text { Witherow } \\
\text { et al. [22] } \\
\text { English }\end{array}$} & \multirow{6}{*}{$\begin{array}{l}\text { Prospec- } \\
\text { tive study }\end{array}$} & \multirow{6}{*}{$\begin{array}{l}\mathrm{n}=216 \\
\text { Arthroscopic ACL recon- } \\
\text { struction middle third of } \\
\text { patella tendon graft }\end{array}$} & \multirow{6}{*}{$\begin{array}{l}\text { Int: rehab pro- } \\
\text { gramme with } \\
\text { CPM starting im- } \\
\text { mediately after } \\
\text { surgery, } 24 \mathrm{~h} 60^{\circ} \\
\text { for } 1 \text { day only } \\
\text { Con: identical re- } \\
\text { hab programme } \\
\text { without CPM }\end{array}$} & \multicolumn{4}{|l|}{ Pain: } \\
\hline & & & & \multicolumn{4}{|c|}{ Mean number of doses intramuscular analgesia } \\
\hline & & & & During hospital stay: & CPM: 2.3 & Con: 1.3 & $\mathrm{p}<0.0001$ \\
\hline & & & & \multicolumn{4}{|l|}{ Pain: } \\
\hline & & & & \multicolumn{4}{|c|}{ Mean number of doses oral analgesia } \\
\hline & & & & During hospital stay: & CPM: 3.6 & Con: 2.6 & $\mathrm{p}<0.0008$ \\
\hline \multirow{10}{*}{$\begin{array}{l}\text { Yates et al. } \\
\text { [23] } \\
\text { English }\end{array}$} & \multirow[t]{10}{*}{ RCT } & \multirow{10}{*}{$\begin{array}{l}\mathrm{n}=30 \\
\text { Arthroscopic ACL recon- } \\
\text { struction, middle third of } \\
\text { patella tendon graft }\end{array}$} & Int: rehab pro- & ROM: Flexion & & & \\
\hline & & & gramme with & Passive knee flexion in & egrees measured on: & & \\
\hline & & & mediately after & Postop day 3 & CPM: 89.9 & Con: 74.4 & $\mathrm{p}<0.05$ \\
\hline & & & surgery, 16 hours & Postop day 7 & CPM: 104.6 & Con: 85.2 & $p<0.05$ \\
\hline & & & $\begin{array}{l}\text { daily during post- } \\
\text { op days } 1-3 \text {, }\end{array}$ & Postop 3 weeks: & CPM: 118.8 & $\begin{array}{l}\text { Con: } \\
112.6\end{array}$ & $p<0.05$ \\
\hline & & & 6 hours daily, for & ROM: Flexion & & & \\
\hline & & & 2 weeks. Initially & Active knee flexion in & grees measured on: & & \\
\hline & & & $0-60^{\circ}$, increasing & Postop day 3 & CPM: 77.0 & Con: 63.5 & $\mathrm{p}<0.05$ \\
\hline & & & & Postop day 7 & CPM: 92.6 & Con: 74.0 & $p<0.05$ \\
\hline & & & $\begin{array}{l}\text { Con: identical re- } \\
\text { hab programme } \\
\text { without CPM }\end{array}$ & Postop 3 weeks: & CPM: 111.1 & $\begin{array}{l}\text { Con: } \\
103.8\end{array}$ & $p<0.05$ \\
\hline
\end{tabular}

Swelling at mid-patella level during the third to seventh postoperative days:

CPM showed no significant effect on swelling at mid-patella level during the third to seventh postoperative days (Hedges' $\mathrm{g}=0.25 ; 95 \% \mathrm{Cl}=-0.54$ to $1.04 ; \mathrm{Q}=6.85 ; \mathrm{df}(\mathrm{Q})=2 ; \mathrm{p}=0.03$; $\mathrm{I}^{2}=71 \%$ ) when compared with rehabilitation without CPM, as shown in > Fig. 6.

Swelling at mid-patella level during the fourth to sixth postoperative weeks: CPM showed significantly beneficial effects on swelling at mid-patella level as measured during the fourth to sixth postoperative weeks (Hedges' $\mathrm{g}=0.77 ; 95 \% \mathrm{Cl}=0.35$ to
1.18; $Q=0.44 ; d f(Q)=2 ; p=0.80 ; I^{2}=0 \%$ ) when compared with rehabilitation without CPM, as shown in > Fig. 7 .

Pain medication during the first 24 hours after surgery: CPM showed a significantly beneficial effect on the need for pain medication during the first 24 hours after surgery (Hedges' $\mathrm{g}=0.93$; $95 \% \mathrm{Cl}=0.41$ to $1.45 ; \mathrm{Q}=0.65 ; \mathrm{df}(\mathrm{Q})=1 ; \mathrm{p}=0.42 ; \mathrm{I}^{2}=0 \%$ ) when compared with rehabilitation without CPM, as shown in $\mathbf{r}$ Fig. 8 .

Number of PCA-button pushes during the first 24 hours after surgery: rehabilitation with CPM showed a significantly smaller number of PCA button pushes by the patients during the first 24 hours after surgery $(M D=31.20 ; 95 \% \quad C l=11.35$ to 51.05 ; 
- Table 2 Risk of bias overview.

\begin{tabular}{|l|l|l|l|l|l|}
\hline & $\begin{array}{l}\text { Sequence } \\
\text { generation }\end{array}$ & $\begin{array}{l}\text { Allocation } \\
\text { concealment }\end{array}$ & $\begin{array}{l}\text { Blinding partici- } \\
\text { pants, personnel }\end{array}$ & $\begin{array}{l}\text { Incomplete } \\
\text { outcome data }\end{array}$ \\
\hline Bizzini et al. [18] & + & $?$ & $?$ & - & $\begin{array}{l}\text { Other sour- } \\
\text { come reportive out- }\end{array}$ \\
\hline Engstrom et al. [13] & $?$ & $?$ & $?$ & + \\
\hline McCarthy et al. [19] & $?$ & $?$ & + & + \\
\hline Noyes et al. [20] & $?$ & $?$ & + & + \\
\hline Rigon et al. [21] & $?$ & $?$ & - & + \\
\hline Terzi et al. [16] & $?$ & $?$ & $?$ & + \\
\hline Witherow et al. [22] & $?$ & $?$ & + & + \\
\hline Yates et al. [23] & + & $?$ & $?$ \\
\hline +: low risk of bias, - high risk of bias, ?: unclear (uncertain risk of bias) & + \\
\hline
\end{tabular}

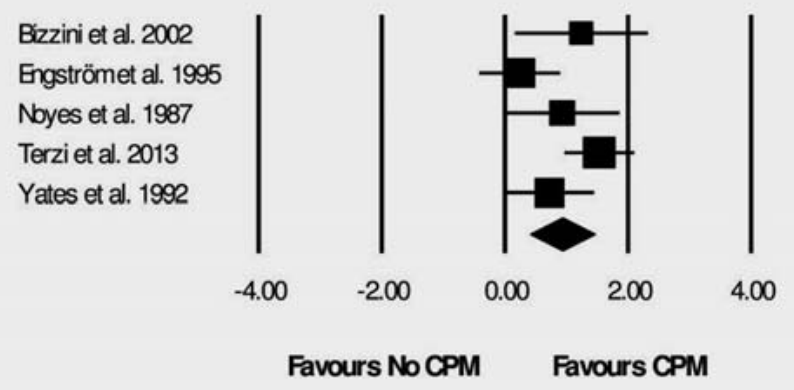

- Fig. 2 Difference in means (95\% Cl): flexion degrees during postop days three to seven by pooling data from four studies $(n=122)$.

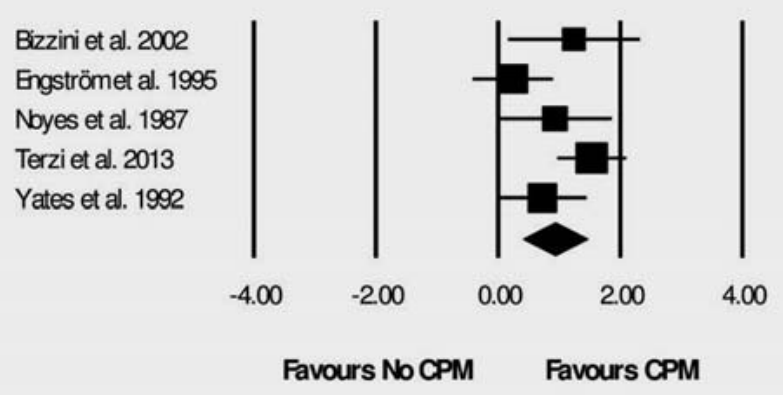

- Fig. 3 Hedges' g $(95 \% \mathrm{Cl})$ of effect of CPM on flexion during postop weeks three to six by pooling data from five studies $(n=156)$.

\section{Bizzini et al. 2002 Nbyes et al. 1987 Terzi et al. 2013}
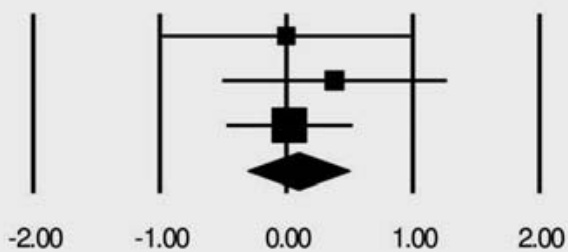

Favours No CPM

\section{Favours CPM}

- Fig. 4 Hedges' g (95\% Cl) of effect of CPM on extension during postop days three to seven by pooling data from three studies $(n=92)$.

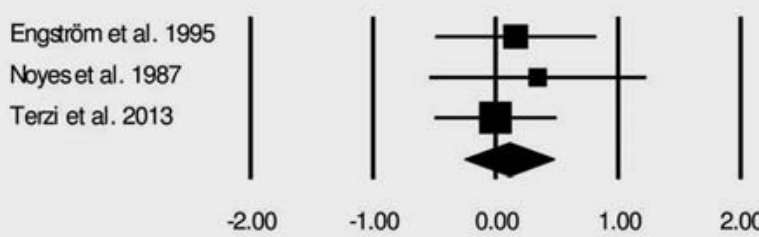

Favours No CPM Favours CPM

- Fig. 5 Hedges' g (95\% Cl) of effect of CPM on extension during postop weeks three to six by pooling data from three studies $(n=112)$. 
Noyeset al. 1987

Rigon et al. 1993

Yateset al. 1992

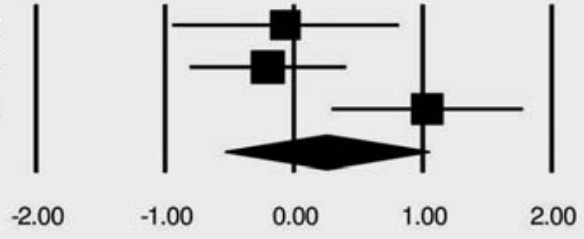

Favours No CPM

Favours CPM

- Fig. 6 Hedges' $\mathrm{g}(95 \% \mathrm{Cl})$ of effect of CPM on swelling at mid patella level between the third and the seventh postoperative days by pooling data from three studies $(n=88)$.

\section{Mocarthy et al.1993
Yateset al. 1992
Fig. 8 Hedges' $g$ (95\% Cl) of effect of CPM on the need for pain
medication during the first 24 hours after surgery by pooling data
from two studies ( $\mathrm{n}=60)$.}

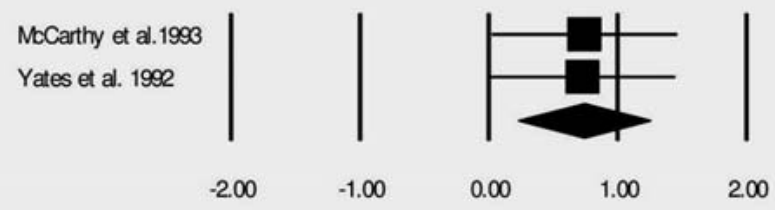

Favours No CPM Favours CPM

- Fig. 10 Hedges' $\mathrm{g}(95 \% \mathrm{Cl})$ of effect of CPM on the need for pain medication on the second postoperative day by pooling data from two studies $(n=60)$.

$Q=0.00 ; d f(Q)=1 ; p=0.93 ; I^{2}=0 \%$ ) when compared with rehabilitation without CPM, as shown in > Fig. $\mathbf{9}$.

Pain medication on the second postoperative day: CPM showed a significantly beneficial effect on the need for pain medication on the second postoperative day (Hedges' $g=0.74 ; 95 \%$

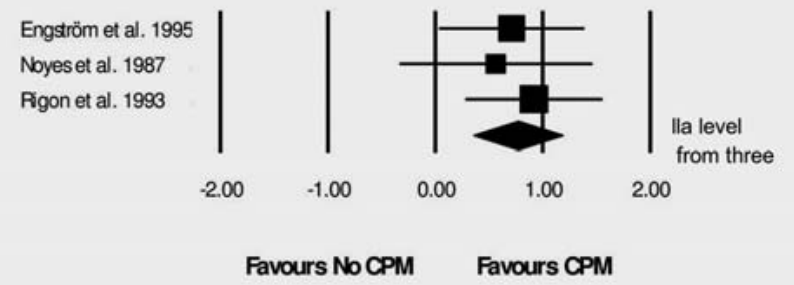

- Fig. 7 Hedges' $\mathrm{g}(95 \% \mathrm{Cl})$ of effect of CPM on swelling at mid patella level as measured between the fourth and the sixth postoperative days by pooling data from three studies $(n=92)$.
Sudy name

McCarthy et al. 1990

Yates et al. 1998

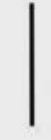

$-60.00$

\section{Difference in means and $95 \% \mathrm{Cl}$}

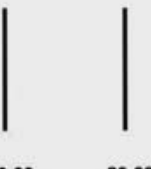

$-30.00$

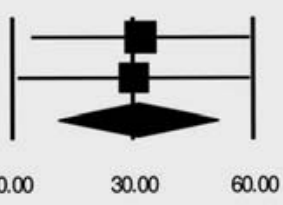

Favours No CPM Favours CPM
- Fig. 9 Difference in means $(95 \% \mathrm{Cl})$ of the number of PCA-button pushes by the patients during the first 24 hours after surgery by pooling data from two studies $(n=60)$.

$\mathrm{Cl}=0.23$ to $1.25 ; \mathrm{Q}=0.00 ; \mathrm{df}(\mathrm{Q})=1 ; \mathrm{p}=0.98 ; \mathrm{I}^{2}=0 \%$ ) when compared with rehabilitation without CPM, as shown in ₹ Fig. 10 .

Pain on the third postoperative day: CPM showed no significant effect on perceived pain on the third postoperative day (Hedges' $\mathrm{g}=0.04 ; 95 \% \mathrm{Cl}=-0.37$ to $0.45 ; \mathrm{Q}=0.35 ; \mathrm{df}(\mathrm{Q})=1 ; \mathrm{p}=0.56$; $\mathrm{I}^{2}=0 \%$ ) when compared with rehabilitation without CPM, as shown in > Fig. 11.

Sensitivity analysis: Range of motion, flexion during the third to sixth postoperative weeks after removal of the study of Engstrom et al. [13]: the effect size shifts from Hedges' $g=0.93$ ( $95 \% \mathrm{Cl}=0.41$ to $1.44 ; \mathrm{Q}=9.12 ; \mathrm{df}(\mathrm{Q})=4 ; \mathrm{p}=0.06 ; \mathrm{I}^{2}=56 \%$, left) to Hedges' $g=1.17(95 \% \mathrm{Cl}=0.77$ to $1.57 ; \mathrm{Q}=3.29 ; \mathrm{df}(\mathrm{Q})=3$; $p=0.35 ; I^{2}=9 \%$, right), as shown in $>$ Fig. 12 .

\section{Discussion}

The aim of this systematic review and meta-analysis was to summarise the results of empirical studies assessing the effects of CPM on rehabilitation after $A C L$ reconstruction and to evaluate their variability. The research question was: what are the effects of continuous passive motion on range of motion, swelling and pain after $A C L$ reconstruction? The research question could be answered as follows. This systematic review and meta-analysis sug- 


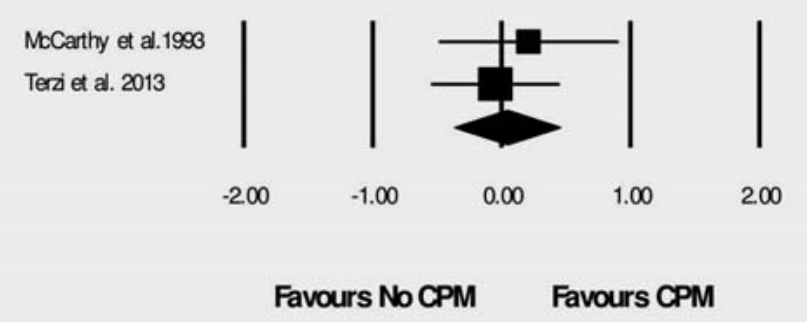

- Fig. 11 Hedges' g $(95 \% \mathrm{Cl})$ of effect of CPM on perceived pain on the third postoperative day by pooling data from two studies $(n=90)$.

gest that the use of continuous passive motion as a part of postoperative treatment after anterior cruciate ligament surgery has some beneficial effects on both short-term outcomes (first seven postoperative days) and long-term outcomes (second to sixth weeks after surgery). As for the short-term outcomes, the analysis determined moderate to strong effect sizes on the need for pain medication during the first two days after surgery. It determined a weighted mean difference of 31 fewer PCA-button pushes during the first 24 hours (a reduction of nearly $50 \%$ ). It also showed an overall weighted mean difference of $11.6^{\circ}$ more knee flexion. Concerning the long-term outcomes, a moderate overall weighted mean effect size was shown for swelling of the knee as measured between the fourth and the sixth postoperative weeks at mid-patella level. A strong overall weighted mean effect size could be identified for knee flexion as measured between the third and the sixth postoperative weeks.

These results contrast with the conclusions of Smith and Davies [6], quoted in the introduction section, who did not appraise CPM as a beneficial treatment after $\mathrm{ACL}$ reconstruction. The reasons for this contrast might be found in the following facts. Smith and Davies [6] published a narrative review, without a meta-analysis. Therefore, they were not able to provide conclusions based on a quantitative analysis. Furthermore, as an inclusion criterion they restricted the publication language to English. Although five of their eight included studies were the same studies that were included in this current meta-analysis [13,19,21-23], three others were not. Smith and Davies [6] additionally included two studies that reported joint stability as the main outcome, which was not of interest in this current meta-analysis. Furthermore, they included one study that did not report any statistical measures of dispersion and therefore was not appropriate for this meta-analysis. On the other hand, in this current meta-analysis three further studies were included: Bizzini et al. [18], who published in German, Terzi et al. [20]. who published in Turkish and Noyes et al. [20] The latter was not included by Smith and Davies [6] for unknown reasons.

However, the results of this current meta-analysis should be considered with caution because the amount of data in this meta-analysis was predominantly small. With eight studies only, counting 14, 18, 30, 30, 34, 40, 60 and 216 participants respec-
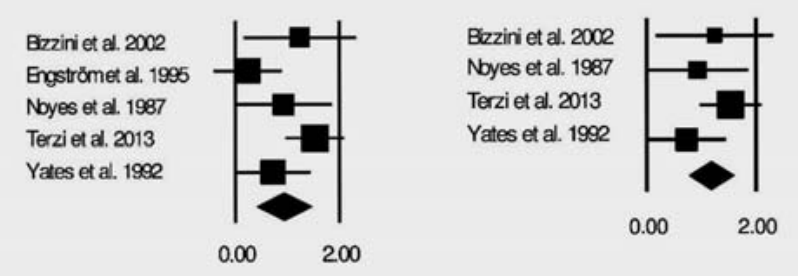

- Fig. 12 Sensitivity analysis: shift of Hedges' g (95\% Cl) after removal of one outlying study.

tively, the number of subjects under investigation was rather limited for a quantitative analysis. In particular, the calculated weighted effect sizes for pain were based on a very small base of only two studies per analysis. The effect sizes of the individual studies for flexion showed a moderate level of inconsistency, as shown by $\mathrm{I}^{2}$-values of $64 \%$ and $56 \%$ respectively.

Six of the eight studies were published before the year 2000 $[13,19-23]$. Before 2000, most non-drug studies were not conducted according to the guidelines for good clinical practice (GCP). Correspondingly low scores for methodological quality had to be given to the studies included in this review, the majority of them showing an unclear risk of bias. Rogan et al. [24] and Jaggi et al. [25] postulated that researchers should follow GCP guidelines, stating that the target of GCP should be to establish and maintain standards of data quality and safety for participants as well as to reduce risk of bias. The main instruments for maintaining high internal validity are allocation concealment, randomisation and blinding [24]. However, in non-drug studies these preconditions cannot always be easily fulfilled. In the context of the current topic, blinding therapists and patients for the application of a continuous passive motion device is not possible (although at least assessors and statisticians could be blinded). Future research is needed that takes the GCP guidelines into account and plans studies with larger sample sizes.

Nevertheless, bearing in mind the limitations of this current review and meta-analysis, our findings seem to indicate that continuous passive motion is applicable in order to reduce pain and swelling and to promote regaining flexion range of motion. This last result is in line with the review of Knapik et al. [10] who found in rabbit studies that CPM improves range of motion of the knee significantly and argued the rabbit model is relevant to humans.

In contrast to the review of Harvey et al. [26], who found that effects of CPM on flexion range of motion in patients after knee total arthroplasty were significant but not clinically meaningful, our findings concerning CPM in patients after $A C L$ reconstruction do seem to be clinically meaningful regarding ROM recovery. If we had decided to calculate the weighted mean difference in passive flexion range of motion in the period after the first postoperative week (which we abandoned on grounds of methodological weak- 
ness), a difference of $13^{\circ}$ of flexion would have resulted, which can be regarded as clinically relevant.

Our post-hoc sensitivity analysis showed that after removing the study of Engstrom et al. [13], who was the only one that had measured knee flexion as late as in the sixth postoperative week and measured it by comparing the operated knee with the sound knee of the same participant, the effect size yet increased from Hedges' $g=0.93$ to Hedges' $g=1.17(95 \% \mathrm{Cl}=0.77$ to 1.57 ; $Q=3.29 ; \mathrm{df}(\mathrm{Q})=3 ; \mathrm{p}=0.35 ; \mathrm{I}^{2}=9 \%$ ), representing a difference in means of $15^{\circ}$ of flexion on a low heterogeneity level.

The effect size of Hedge's $g=0.77$ for swelling of the knee between the fourth and the sixth postoperative weeks becomes more understandable if we try to convert it to a physically imaginable unit. If we had calculated MD instead of Hedges' g, a mean circumference reduction at mid-patella level of $0.6 \mathrm{~cm}$ would have resulted. Assuming a normal circumference of $40 \mathrm{~cm}$ and calculating the cross-sectional area of the knee as $\pi r^{2}$ after deriving $r$ from $2 \pi r=40 \mathrm{~cm}$ ), this would mean a cross-sectional area reduction of approximately $4 \mathrm{~cm}^{2}$ at mid-patella level. This should be regarded as clinically relevant.

For the significant effects on knee flexion as measured between the third and the sixth postoperative weeks, no publication bias could be identified. The classic fail-safe $\mathrm{N}$ test showed that 34 further nonsignificant or negative studies would be needed to bring its $\mathrm{p}$-value above 0.05 . No studies were trimmed in the trim and fill test. For the other outcomes, unfortunately no funnel plots could be created as the minimum amount of required studies was not achieved.

Further limitations of this review should be discussed. Firstly, the conclusions are based on studies that showed inconsistency in the application modalities of continuous passive motion, varying from one hour daily [16] to sixteen hours daily [19]. The total number of intervention days also varied widely from only one day [22] to two weeks [23]. The number of available studies was too small for a subgroup or covariate analysis which might have further analysed the influence of the differences in applied dosage, from which recommendations for clinicians regarding dosage efficacy could have been derived. Secondly, not all studies reported all the required information and the meta-analysis had to be managed with surrogate procedures as described in the manual of the Cochrane Handbook for Systematic Reviews of Interventions [14], for example when integrating the outcomes of the study of Rigon et al. [21], as described in the Method section. However, a posthoc sensitivity analysis showed that replacing the surrogate standard deviations of the Rigon et al. study [21] with higher or lower values (double and half, respectively) did not change the effect sizes of the meta-analysis concerning swelling in a meaningful way. The nonsignificant difference in swelling in the first week (Hedges' $\mathrm{g}=0.25 ; 95 \% \mathrm{Cl}=-0.54$ to 1.04 ) would shift to a still non-significant Hedges' $\mathrm{g}=0.18(95 \% \mathrm{Cl}=-0.72$ to 1.07$)$ by halving the chosen standard deviation, or shift to another nonsignificant Hedges' $g=0.29$ (95\% Cl $=-0.45$ to 1.03 ) by doubling it. On the other hand, the significant difference in swelling in the fourth to the sixth week s (Hedges' $\mathrm{g}=0.77 ; 95 \% \mathrm{Cl}=0.35$ to 1.18 ) remained significant after halving the surrogate standard deviation for Rigon et al. [21] (Hedges' $\mathrm{g}=1.05 ; 95 \% \mathrm{Cl}=0.26$ to 1.85 ) or doubling it (Hedges' $\mathrm{g}=0.57 ; 95 \% \mathrm{Cl}=0.16$ to 0.98 ).
In order to analyse the effects of continuous passive motion after $\mathrm{ACL}$ reconstruction more precisely, further research should be recommended. In particular, the relation between the effects of CPM and its dosage should be investigated in further randomised controlled trials of high quality as a basis for further conclusions about the value of CPM in the postoperative treatment after ACL surgery.

\section{Conclusion}

This review provides low-level evidence supporting the use of CPM after $A C L$ reconstruction. It indicates that CPM might be helpful in postoperative pain management (short term), in regaining flexion ROM (short and long term) and in swelling reduction (long term). We recommend clinicians continue the application of CPM after $\mathrm{ACL}$ reconstruction and attentively monitor future research into this topic.

Future high quality RCTs are needed to confirm the findings of this review and to find out more about efficacy and efficiency of dosage parameters of CPM application after ACL reconstruction.

\section{Data Access}

The statistical analysis plan is available on request from the corresponding author.

\section{Funding Statement}

This study has no funding.

Danksagung

We are grateful to Ingrid B. Müller and Anne Pfeiffer for database search support, to Marius Jaspers and Bennie Ross for English writing support and to Jon Lory for providing time

\section{Conflict of Interest}

The authors declare that they have no conflict of interest.

\section{References}

[1] Salter RB, Bell RS, Keeley FW. The protective effect of continuous passive motion on living articular cartilage in acute septic arthritis: an experimental investigation in the rabbit. Clin Orthop Relat Res 1981; 159: 223-247

[2] O'Driscoll SW, Kumar A, Salter RB. The effect of continuous passive motion on the clearance of a hemarthrosis from a synovial joint: an experimental investigation in the rabbit. Clin Orthop Relat Res 1983; 176: 305

[3] Salter RB. The biologic concept of continuous passive motion of synovial joints. The first 18 years of basic research and its clinical application. Clin Orthop Relat Res 1989; 242: 12-25

[4] Salter RB, Hamilton HW, Wedge JH et al. Clinical application of basic research on continuous passive motion for disorders and injuries of synovial joints: a preliminary report of a feasibility study. J Orthop Res 1983 1: 325-342

[5] O'Meara PM, O'Brien WR, Henning CE. Anterior cruciate ligament reconstruction stability with continuous passive motion. The role of isometric graft placement. Clin Orthop Relat Res 1992: 201-209 
[6] Smith TO, Davies L. The efficacy of continuous passive motion after anterior cruciate ligament reconstruction: a systematic review. Phys Ther Sport 2007; 8: 141-152

[7] Lobb R, Tumilty S, Claydon LS. A review of systematic reviews on anterior cruciate ligament reconstruction rehabilitation. Phys Ther Sport 2012; 13: $270-278$

[8] Egger M, Zellweger-Zähner T, Schneider M et al. Language bias in randomised controlled trials published in English and German. Lancet 1997; 350: 326-329

[9] Higgins JPT, Green S. Cochrane handbook for systematic reviews of interventions version 5.1.0. The Cochrane Collaboration 2011. Online: https://handbook-5-1.cochrane.org/; last access: 11.09.2018

[10] Knapik DM, Harris JD, Pangrazzi G et al. The basic science of continuous passive motion in promoting knee health: a systematic review of studies in a rabbit model. Arthroscopy 2013; 29: 1722-1731

[11] Shoemaker S, Skyhar M, Simmons T. Orthopaedic rehabilitation. 2nd ed. London: Churchill Livingstone; 1992

[12] Moher D, Liberati A, Tetzlaff J et al. Preferred reporting items for systematic reviews and meta-analyses: the PRISMA statement. Int J Surg 2010; 8: $336-341$

[13] Engström B, Sperber A, Wredmark T. Continuous passive motion in rehabilitation after anterior cruciate ligament reconstruction. Knee Surg Sports Traumatol Arthrosc 1995; 3: 18-20

[14] Higgins JPT, Green S, eds. Cochrane Handbook for Systematic Reviews of Interventions. New Jersey, USA: John Wiley \& Sons, Inc.; 2011

[15] Higgins JP, Thompson SG, Deeks JJ et al. Measuring inconsistency in meta-analyses. BMJ 2003; 327: 557-560

[16] Terzi R, Gultekin A, Atlihan D. The effect of continuous passive motion implementation after anterior cruciate ligament reconstruction. Turkiye Fiziksel Tip ve Rehabilitasyon Dergisi 2013; 59: 314-318
[17] Porzig F, Krüger-Franke M, Kugler A et al. Effekte der CPM-Behandlung nach operativ versorgter isolierter vorderer Keuzbandruptur. SportOrthopädie-Sporttraumatologie 2007; 23: 193-198

[18] Bizzini M, Caporaso F, Drobny T et al. Post-operative rehabilitation following anterior cruciate ligament reconstruction - active vs. passive movement device [German]. Krankengymnastik 2002; 54: 740-744

[19] McCarthy MR, Yates CK, Anderson MA et al. The effects of immediate continuous passive motion on pain during the inflammatory phase of soft tissue healing following anterior cruciate ligament reconstruction. J Orthop Sports Phys Ther 1993; 17: 96-101

[20] Noyes FR, Mangine RE, Barber S. Early knee motion after open and arthroscopic anterior cruciate ligament reconstruction. Am J Sports Med 1987; 15: 149-160

[21] Rigon A, Viola R, Lonedo F. Continuous passive motion in reconstruction of the anterior cruciate ligament. J Sport Traumatol 1993; 15: 187-192

[22] Witherow GE, Bollen SR, Pinczewski LA. The use of continuous passive motion after arthroscopically assisted anterior cruciate ligament reconstruction: help or hindrance? Knee Surg Sports Traumatol Arthrosc 1993; 1: 68-70

[23] Yates CK, McCarthy MR, Hirsch HS et al. Effects of continuous passive motion following $\mathrm{ACL}$ reconstruction with autogenous patellar tendon grafts. J Sport Rehabil 1992; 1: 121-131

[24] Rogan S, Hilfiker R, Schenk A et al. Effects of whole-body vibration with stochastic resonance on balance in persons with balance disability and falls history - a systematic review. Res Sports Med 2014; 22: 294-313

[25] Jaggi U, Joray CP, Brulhart Y et al. [Injuries in the Martial Arts ]udo, Taekwondo and Wrestling - A Systematic Review]. Sportverletz Sportschaden 2015; 29: 219-225

[26] Harvey LA, Brosseau L, Herbert RD. Continuous passive motion following total knee arthroplasty in people with arthritis. The Cochrane Database Syst Rev 2010; (2): CD004260 